\title{
Freeform quad-based kirigami
}

\author{
CAIGUI JIANG, KAUST \\ FLORIAN RIST, KAUST, TU Wien \\ HELMUT POTTMANN, KAUST, TU Wien \\ JOHANNES WALLNER, TU Graz
}

\begin{abstract}
Kirigami, the traditional Japanese art of paper cutting and folding generalizes origami and has initiated new research in material science as well as graphics. In this paper we use its capabilities to perform geometric modeling with corrugated surface representations possessing an isometric unfolding into a planar domain after appropriate cuts are made. We initialize our box-based kirigami structures from orthogonal networks of curves, compute a first approximation of their unfolding via mappings between meshes, and complete the process by global optimization. Besides the modeling capabilities we also study the interesting geometry of special kirigami structures from the theoretical side. This experimental paper strives to relate unfoldable checkerboard arrangements of boxes to principal meshes, to the transformation theory of discrete differential geometry, and to a version of the Gauss theorema egregium.
\end{abstract}

CCS Concepts: • Computing methodologies $\rightarrow$ Shape modeling; Optimization algorithms.

Additional Key Words and Phrases: Kirigami, discrete differential geometry, quad meshes, digital fabrication, computational origami

\section{ACM Reference Format:}

Caigui Jiang, Florian Rist, Helmut Pottmann, and Johannes Wallner. 2020. Freeform quad-based kirigami. ACM Trans. Graph. 39, 6, Article 209 (December 2020), 11 pages. https://doi.org/10.1145/3414685.3417844

\section{INTRODUCTION}

Folding flat sheets of paper along straight creases is called origami, which famously is both a traditional craft and a medium of art. It has attracted quite a lot of attention in Mathematics, Computer Science, and lately also Material Science. A treatment from the viewpoint of computational geometry and algorithms is contained in the textbook [Demaine and O'Rourke 2007]. Generalizing origami, the term kirigami refers to the traditional Japanese art of papercutting, which combines folding with incisions along straight edges or even cutting out holes. Like origami, it has a long history and the word nowadays is employed without the original cultural context; in this paper we use it to indicate the making of structures by cutting and subsequent folding of flat sheet material.

Both origami and kirigami are of great interest for material science because of their capability of modeling microstructures. An important question asked here is the relation between the local

Authors' addresses: Caigui Jiang, KAUST; Florian Rist, KAUST, TU Wien; Helmut Pottmann, KAUST, TU Wien; Johannes Wallner, TU Graz.

Permission to make digital or hard copies of all or part of this work for personal or classroom use is granted without fee provided that copies are not made or distributed for profit or commercial advantage and that copies bear this notice and the full citation on the first page. Copyrights for components of this work owned by others than the author(s) must be honored. Abstracting with credit is permitted. To copy otherwise, or republish, to post on servers or to redistribute to lists, requires prior specific permission and/or a fee. Request permissions from permissions@acm.org.

(C) 2020 Copyright held by the owner/author(s). Publication rights licensed to ACM 0730-0301/2020/12-ART209 \$15.00

https://doi.org/10.1145/3414685.3417844

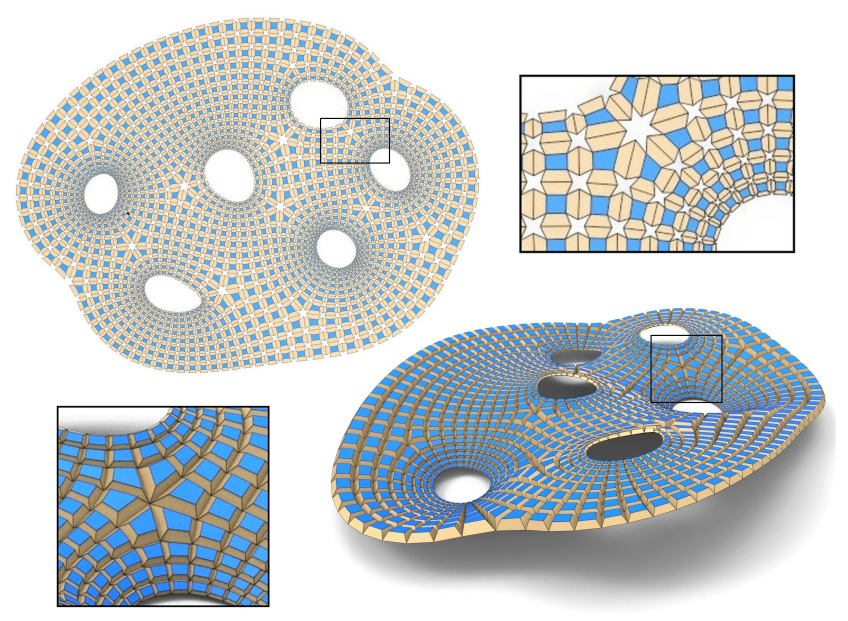

Fig. 1. Closing all star-shaped holes in a 2D domain by folding along prescribed edges produces a corrugated watertight box kirigami surface representation. It is initialized from an orthogonal network of curves on a smooth design surface, and is computed by global optimization.

geometry of the structure and its mechanical properties, see e.g. [Seffen 2012; Wei et al. 2013]. Kirigami structures in particular can be seen as mechanical metamaterials exhibiting auxetic behaviour. This refers to the counterintuitive property of expansion under tensile forces.

The work presented in this paper is about patterns of cuts and folds in a 2D domain which exhibit a certain degree of combinatorial regularity. In fact our patterns are governed by quad meshes which are combinatorially regular except for a limited number of extraordinary vertices, see Fig. 1 . We want to fold the planar pattern into a three-dimensional structure which follows a design surface such that the original holes close - we aim at watertight kirigami representations of free forms.

This is not the only way surfaces can be represented by kirigami structures. For instance, the local combinatorial symmetries of the cut and fold pattern might be those of a hexagonal lattice rather than those of an orthogonal grid. Instead of closing cuts via deployment as we do, one can also open them. Opening cuts to the maximum extent possible even is a way of eliminating unwanted degrees of freedom in deployment, just as closing holes is in our case. For a detailed discussion of prior work we refer to $§ 2$ below.

There is generally a great variety of potential kirigami structures. One motivation to investigate this particular class is fairness and the fact that cuts and folds are based on smooth curve networks, as explained below. Other reasons are efficient use of material (see 

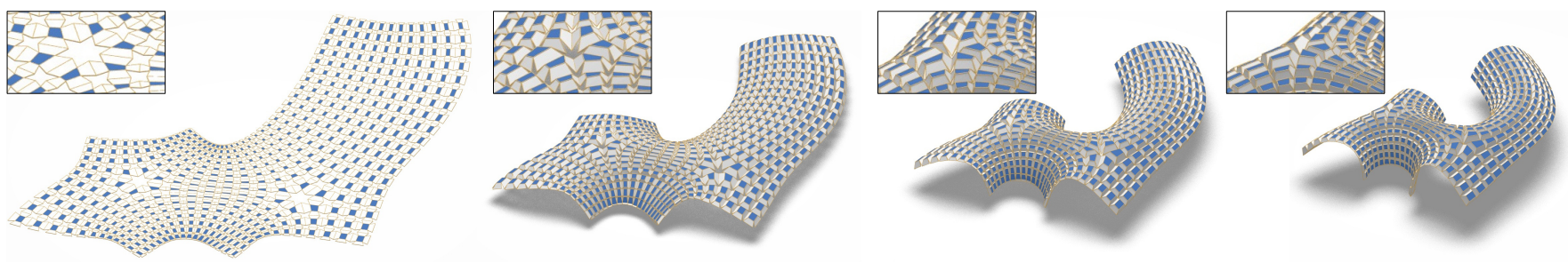

Fig. 2. A folding sequence of a freeform box kirigami structure. The spatial state $M$ is at right, the unfolded planar state $\bar{M}$ is at left.

Figures 14, 15), and a solid connection between individual elements (e.g., compared to the auxetic structures mentioned above).

The structures treated in this paper besides their interesting geometry can be used directly for applications. Rigid and stable kirigami objects of smaller size can be made from paper, sheet metal or acrylic glass. Folding from flat sheets is the very method of fabrication. At a larger scale our work is applicable to modules to be assembled later, e.g. for a suspended ceiling like in Fig. 24. We do not claim that folding is a viable method of fabrication for buildings at the architectural scale, but we point out the use of folding as a distinct design language, as exemplified by the recent Théâtre de Vidy in Lausanne, Switzerland.

\subsection{Contributions}

The main goal of this paper is to provide preliminary results and research. Some important questions, in particular on existence, are left open. The contributions of this paper are the following.

- We experimentally solve the inverse design problem for several classes of watertight kirigami structures with quad combinatorics, each offering different degrees of flexibility. For the most flexible structures, the user can design the appearance of the $2 \mathrm{D}$ pattern together with the resulting 3D structure.

- We work with optimization, initialized by mappings from the design surface to a 2D domain whose principal distortions are being controlled. We show how to compute such mappings (in general, not conformal) between so-called base meshes and how to derive kirigami structures from them.

- We outline relations to discrete differential geometry, in particular to principal meshes and Combescure transformations. We also show how Gauss curvature can be read off the 2D unfolding of kirigami structures.

- By allowing curved cuts, we extend our constructions to the case of kirigami with rounded edges.

- Due to the nonlinear and non-convex nature of the optimization tasks we encounter, success of our procedures cannot be guaranteed mathematically. Results can however be verified a posteriori. We also check the feasibility of our computational models via accompanying physical models.

- The main contribution of this paper is to identify kinds of kirigami that allow systematic initialization and subsequent computation.

\section{PREVIOUS WORK}

Compared to origami, the mathematical and algorithmic treatment of shapes obtainable by both cutting and folding flat sheets is more recent. For example, Castle et al. [2014; 2016] treat the regular 2D hexagonal lattice and explore rule sets for cutting and folding. Kirigami with rotational symmetry are studied by Wang et al. [2017], while cut and fold patterns in general are explored from the viewpoint of mesostructures / material science by Zhang et al. [2015]. Actuation of small kirigami elements and of periodic patterns derived from them has been studied by Tang et al. [2019].

A very important task to be solved in connection with both origami and kirigami is the backward problem, i.e., designing a cut and fold pattern which will deploy into a prescribed threedimensional shape. It also comes under the heading of programming curvature by means of the pattern imposed.

For the folding-only case, which is not the subject of the present paper, we only mention a few examples: Miura-ori type patterns on flat sheets and their generalizations can be folded to assume certain curved forms [Dudte et al. 2016; Tachi 2009]. "Waterbomb" folding patterns can be used to construct actuated tube-like structures [Mukhopadhyay et al. 2020]. It is not surprising that dispensing with cutting and allowing only folding makes the backward problem highly constrained. This can also be observed in the case of folding along curved creases studied by Jiang et al. [2019a]. Relevant to the present paper, Tachi [2010b] proposed a method of approximating any surface by means of origami, which is implemented as the "origamizer" software and is also the subject of the follow-up papers [Demaine and Tachi 2017; Tachi 2010a]. This method identifies parts of a 2D domain that cannot contribute to a desired 3D shape and tucks them away. There are two major differences to our method. One is our emphasis on fairness, which has not been addressed so far in the pure origami case. Thus the results obtained via "origamizer" are not related (as ours are) to special parametrizations of surfaces. The other difference is that we do not need 180 degree folds, even for such special kirigami that constitute seamless surfaces with tucked-away material. This helps real-world applications.

For kirigami constructed by means of both cutting and folding, the backward problem was addressed by Konaković et al. [2016]. They employ conformal geometry to model flat sheet material with small incisions along line segments whose combinatorics is that of a bamboo weaving pattern (hexagonal kagome pattern). These incisions allow the material to expand with a point-dependent scaling factor, and to assume free forms. Follow-up work, likewise based on conformal geometry, treats the process of deployment of a more general class of cut/fold patterns. [Konaković-Luković et al. 2018] construct structures that are fully deployed exactly when the limit of expansion is reached. The class of possible shapes achievable in this way includes nonconvex ones and is only restricted by the 

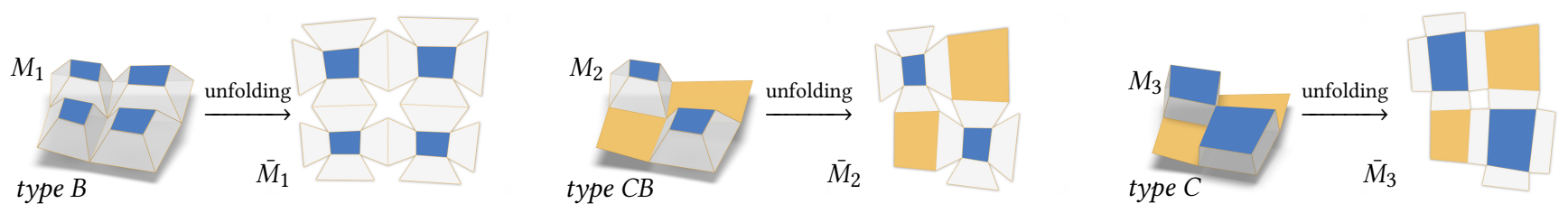

Fig. 3. Different types of kirigami structures $M_{j}$ and their unfolding $\bar{M}_{j}$. From left: Box (B) kirigami $M_{1}$; checkerboard-box (CB) kirigami $M_{2}$; checkerboard (C) kirigami $M_{3}$. Both the spatial arrangement and the unfolding must exist for a true kirigami. Any node in the kirigami structure where $n$ boxes meet corresponds to a $2 n$-sided hole in $\bar{M}$. Typically a B kirigami has 8 -sided holes, a CB kirigami has 4 -sided kite-shaped holes, and a C kirigami has rhombuses.

condition that the method of deployment works only for surfaces where mean curvature does not change sign. We point to a major difference between our kirigami structures and those of Konakovic et al.: Theirs consist of elements connected only at single vertices, which may be problematic for manufacturing.

Choi et al. [2019] start from the regular square grid, deform it, and cut along edges. This allows deployment to checkerboard patterns formed by the original quads alternating with quadrilateral holes. They generate both 2D patterns and patterns following surfaces in 3D by optimizing the above-mentioned deformation.

The contributions mentioned above are, loosely speaking, concerned with a correspondence between a flat domain and a surface in space which is expanding. That expansion is realized by the opening of holes cut into the planar unfolding. In this paper we consider the reverse, namely creating watertight surfaces by the closing of holes.

Previous work in that direction includes [Guseinov et al. 2017]. They do not consider cutting and folding in the sense of kirigami, but cut shapes into small pieces and arrange them on an elastic sheet whose release recreates the shape. The computational problem here is tile layout. This principle was much extended in work by Guseinov et al. [2020] on shells that self-actuate in a controlled way over time.

Watertight kirigami representations of surfaces are explored by Sussmann et al. [2015] who cut holes into a regular hexagonal lattice which is subsequently folded in the shape of prescribed discrete height fields over the original base lattice.

Xie et al. [2015] study in detail the geometry of cube-type and eggbox-type patterns and are able to create kirigami structures which follow special surfaces. Apart from the lack of generality in the shapes that can be achieved, this is the previous work which is closest to our own.

\section{GEOMETRY OF KIRIGAMI STRUCTURES}

This chapter discusses geometric properties of kirigami structures. Their different kinds are introduced in §3.1, where also the relation to curve networks on surfaces is presented. This forms the geometric basis of the initialization procedures of $\S 4.1$. $\$ 3.2$ discusses the degrees of freedom in the construction of kirigami structures, which has direct implications on the solvability of the optimization tasks of $\S 4.2$. Sections 3.3 and 3.4 on relations to differential geometry do not concern the algorithmic part of this paper.

\subsection{Quad-mesh based kirigami}

The kirigami structures treated in this paper and their unfolding can both be modelled as quad meshes - see Fig. 3 which is in detail discussed below. It is convenient to use a mesh $M$ representing the spatial structure that is combinatorially equivalent to the mesh $\bar{M}$ representing its unfolding, even if in $M$ many vertices coincide. We symbolically write $\bar{M}=(\bar{V}, \bar{E}, \bar{F}) \stackrel{\text { folding }}{\longrightarrow} M=(V, E, F)$.

The unfolding $\bar{M}$ is contained in the plane $\mathbb{R}^{2}$. The deployment mapping (folding) is isometric when restricted to faces, see Fig. 2. The holes occurring in $\bar{M}$ will be closed in the final kirigami structure M.

Our description of different types of kirigami starts by defining a box as a polyhedral surface which has the combinatorics of a cube

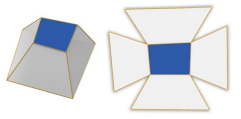
with one face removed. We require that the remaining 5 faces are planar, but the edges around the missing face do not have to lie in a common plane. Such a box can be isometrically flattened to a plane after cutting along the four edges emanating from the boundary. The requirement of planarity is necessary, if we want manufacturing by folding flat sheets along straight lines - see the appendix.

Box (B) kirigami. We start from a quadrilateral base mesh $B_{M}$, where we replace all faces by boxes glued in instead. Boxes are cut open as described above. If the result can be isometrically unfolded into the plane (valence $n$ vertices of $B_{M}$ opening up to form $2 n$ sided holes), this arrangement is called a box kirigami structure see Fig. 3, left.

We wish to relate the base mesh to a curve network on a smooth surface, and the kirigami structure is seen as a corrugated watertight approximation of that smooth surface. Therefore it is understood that most vertices of $B_{M}$ have valence 4 , with the exception of a small number of extraordinary vertices, cf. Fig. 1.

Checkerboard-Box (CB) kirigami. The box kirigami introduced in the previous paragraph can be modified and specialized in different ways. One is the following. If the base quad mesh $B_{M}$ is coloured in a checkerboard pattern and only faces of one color are replaced by boxes, the result is a checkerboard-box kirigami structure, provided an isometric unfolding exists, cf. Fig. 3, center.

A valence $n$ vertex of the base mesh is contained in $n / 2$ boxes and unfolds to a hole with $n$ sides (the checkerboard property implies that $n$ is even). The typical value is $n=4$. Since unfolding is isometric, this quadrilateral hole has the shape of a kite.

Checkerboard (C) kirigami. We define checkerboard kirigami by additionally requiring that boxes share an edge whenever they are adjacent, see Fig. 3, right. Such kirigami are highly constrained. In this paper we show their existence only by numerical experiments. 


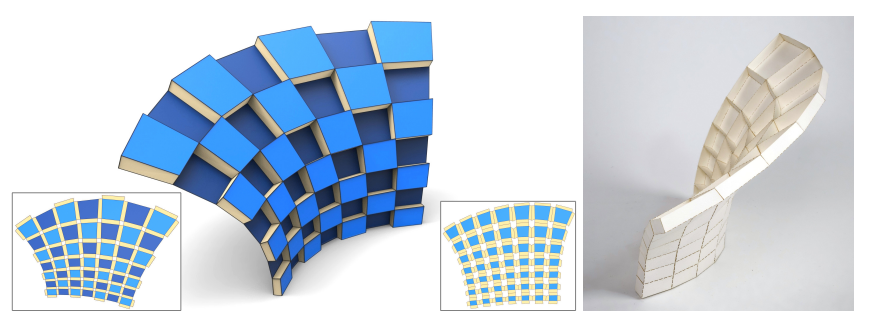

Fig. 4. Highly constrained box kirigami: Type $\mathrm{C}$ kirigami (left) and support structure kirigami, both shown with unfolding, require neighbouring boxes to fit seamlessly together. They thus contain discrete-developable surfaces transverse to the base mesh $B_{M}$. If boxes emerge from $B_{M}$ approximately orthogonally, then the edges of $B_{M}$ follow principal curvature lines.

Further kinds of kirigami structures. The basic principle of box kirigami can be modified and specialized in further ways. For example, boxes could be added not just on one side of the base mesh but on both. For any box whose base is planar, we can apply a reflection to move it to the other side of the base mesh without changing the unfolding. Figure 7, center, shows an example where additional constraints have been imposed: Here the two faces incident with any edge of the base mesh are forced to lie in a common plane, thus creating flat hexagons.

Other specializations are pyramid-shaped boxes defined by the top face shrunk to a point (Fig. 20). An entirely different kind of requirement is that boxes incident with a base mesh vertex are to share an edge, see Fig. 4. This yields almost a support structure in the sense of Pottmann et al. [2007]. Its existence is only shown by numerical experiments.

\subsection{Existence of quad-mesh based kirigami}

While type B kirigami exhibit quite a lot of degrees of freedom, kirigami of $\mathrm{CB}$ type are more constrained. In the following we give a detailed description of their geometric properties, and we also count their degrees of freedom.

Figure 5 shows both the unfolded and the spatial state of a small CB kirigami structure consisting only of the 1-neighbourhood of a 4sided hole. We call this a node, and we introduce angles $\alpha_{i}, \beta_{i}, \gamma_{i}, i=$
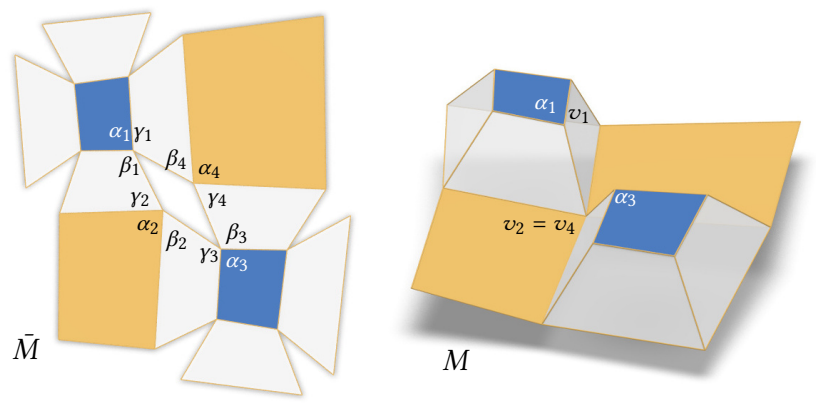

Fig. 5. Folding a node in a CB kirigami. If all faces are thought rigid, the closed form of the node consists of four rigid bodies ( 2 boxes, 2 flat quadrilaterals) connected with hinges and thus is flexible with 1 degree of freedom. The angles $\alpha_{k}, \beta_{k}, \gamma_{k}$ around the 4 -sided hole play a role in our d.o.f. count.

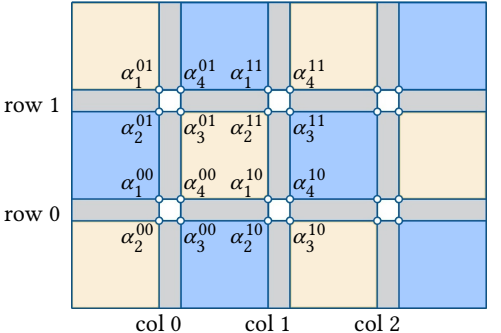

Fig. 6. Unfolding a combinatorially regular CB kirigami. In row $i$ and column $j$ four vertices $\bar{v}_{k}^{i j}$ form the 4-sided hole whose closure defines the folding. Faces incident to $\bar{v}_{k}^{i j}$ have angles $\alpha_{k}^{i j}, \beta_{k}^{i j}, \gamma_{k}^{i j}$, see Fig. 5

$1, \ldots, 4$ at vertices $\bar{v}_{1}, \bar{v}_{2}, \bar{v}_{3}, \bar{v}_{4}$ (which are not labelled in the figure). The folding of boxes is possible only if appropriate edgelengths of the 4-sided hole are equal.

To be precise, the two edges emanating from $v_{i}$ must have the same length, and so do the edges emanating from $v_{i+2}$ (indices modulo 4), where either $i=1$ (this is the case in Fig. 5) or $i=2$. Consequently, one of the two following equalities must hold:

$$
\begin{aligned}
& \text { boxes at } \bar{v}_{1}, \bar{v}_{3}: \Longrightarrow\left|\bar{v}_{1} \bar{v}_{2}\right|=\left|\bar{v}_{1} \bar{v}_{4}\right|,\left|\bar{v}_{3} \bar{v}_{2}\right|=\left|\bar{v}_{3} \bar{v}_{4}\right|, \\
& \text { boxes at } \bar{v}_{2}, \bar{v}_{4}: \Longrightarrow\left|\bar{v}_{2} \bar{v}_{3}\right|=\left|\bar{v}_{2} \bar{v}_{1}\right|,\left|\bar{v}_{4} \bar{v}_{1}\right|=\left|\bar{v}_{4} \bar{v}_{3}\right| .
\end{aligned}
$$

Obviously, (1) ensures that under the assumption of rigid faces and edges acting as hinges, we can close the hole. With the hole closed, the node consists of 4 rigid bodies moving in the manner of a spherical 4-bar linkage, with 1 degree of freedom, see Fig. 5.

3.2.1 Existence of checkerboard box (CB) kirigami. We now discuss the construction of larger $\mathrm{CB}$ kirigami by extending the node around a single hole which is shown by Fig. 5 . We assume a combinatorially regular unfolding as in Fig. 6, with vertices $\bar{v}_{k}^{i j}$ of the 4-sided hole at position $(i, j)$, angles $\alpha_{k}^{i j}$ and so on.

- We assume that the node (the 1-neighbourhood of the hole) in position $(0,0)$ has already been constructed and try to proceed by induction.

- For the construction of vertices $\bar{v}_{k}^{10}$, observe that $\bar{v}_{1}^{10}, \bar{v}_{2}^{10}$ as well as the angle $\alpha_{2}^{10}$ already occur in the $(0,0)$ node. By rigidity of boxes, also $\beta_{2}^{10}$ is already defined. Otherwise we can choose the $(1,0)$ node arbitrarily, only subject to condition (1). After folding it becomes a mechanism, indeed a spherical 4-bar linkage (cf. Fig. 5, right), whose degree of freedom is used to join it to the $(0,0)$ node.

- Propagation to position $(0,1)$ is analogous. Angles $\alpha_{2}^{01}$ and $\gamma_{2}^{01}$ are determined by the $(0,0)$ node.

- Propagation to position $(1,1)$ meets an obstacle. Since angles $\alpha_{2}^{11}, \beta_{2}^{11}, \gamma_{2}^{11}$ are determined by nodes at $(1,0)$ and $(0,1)$, the shape of the hole at position $(1,1)$ is determined, and the kite condition (1) acts as a global constraint on the entire previous construction. Having folded the $(1,1)$ node into space, we need to operate its 1parameter flexion to join it with both neighbours. Obviously, we are 1 d.o.f. short.

This shows that we can construct a full CB kirigami structure if we can meet 2 conditions every time a node is added (fewer at the boundary). Forcing the structure to follow a reference surface amounts to another condition per forward step. On the other hand, every time we propagate, we add 3 vertices with a total of 6 d.o.f. (more at the boundary). 

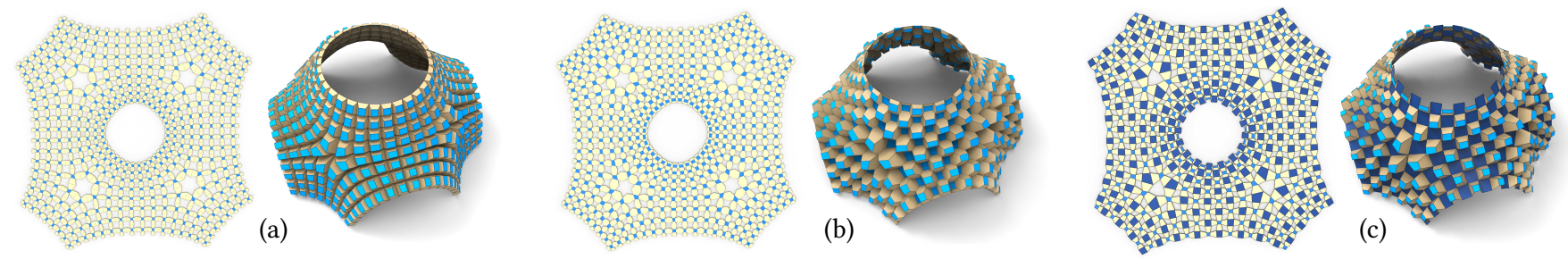

Fig. 7. Different kinds of Box kirigami based on the same network of curves on the reference surface, here the network of principal curvature lines. We show both the unfolding and the final state (not to scale). (a) a general box kirigami structure, (b) a kirigami structure where boxes are lying on both sides of the base mesh in an alternating way. We additionally impose the condition that for each edge of the base mesh, the two adjacent faces, each belonging to the side of a box, must form a planar hexagon. Note that the unfolding does not significantly differ from the first example. (c) A type CB kirigami.

Thus, CB kirigami cannot be constructed inductively by propagation, but rather by solving a system of constraints. It is reassuring that there are twice as many variables as constraints.

3.2.2 Existence of checkerboard kirigami. Those are a specialization of $\mathrm{CB}$ kirigami. Continuing the d.o.f. count from the previous paragraph, in each propagation step we need 3 d.o.f. for the constraint that neighbouring boxes have a common edge. This implies that the construction of $\mathrm{C}$ kirigami is considerably harder than CB kirigami, and proper initialization of optimization becomes important. Our numerical experiments indicate that $\mathrm{C}$ kirigami exist.

\subsection{Differential geometry of checkerboard kirigami}

Checkerboard (type C) kirigami structures exhibit some interesting geometry. Imposing fairness as an optimization goal, we see our structures as discretizations of parametric surfaces including derivatives.

3.3.1 Relations to principal parametrizations of surfaces. Each edge of the base mesh $B_{M}$ contains a face of a box in the kirigami structure $M$, and we typically require that those faces are approximately orthogonal to $B_{M}$.

For a succession of edges along a mesh polyline in $B_{M}$, the abovementioned faces of boxes constitute a discrete developable surface along that mesh polyline, implying that the base mesh $B_{M}$ discretizes a principal parametrization [Bobenko and Suris 2009]. This has an important consequence for initialization of our optimization procedure: The base mesh must follow principal curvature lines of a surface.

Note that this follows already from the requirement that the boxes involved in the checkerboard kirigami are approximately orthogonal to the base mesh $B_{M}$. The fact that mesh polylines in $B_{M}$ must be approximately orthogonal to each other, as well as faces are approximately flat, are consequences of it.
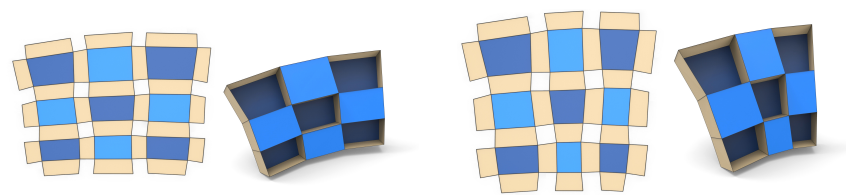

Fig. 8. A pair of edgewise parallel type $C$ kirigami structures with their respective unfoldings. The property of existence of an isometric unfolding is invariant w.r.t. such Combescure transforms.
3.3.2 Combescure transforms. Note that in an unfolding of a type $C$ kirigami, all edges of a hole have the same length. For a 4-sided hole as in Fig. 5 this means

$$
\begin{aligned}
& \alpha_{1}+\beta_{1}+\gamma_{1}=\alpha_{3}+\beta_{3}+\gamma_{3}=: \delta_{13}, \\
& \alpha_{2}+\beta_{2}+\gamma_{2}=\alpha_{4}+\beta_{4}+\gamma_{4}=: \delta_{24}, \quad \delta_{13}+\delta_{24}=3 \pi .
\end{aligned}
$$

The last equation follows from the angle sum $2 \pi$ of quadrilaterals. Obviously (2) implies that the hole is a rhombus, meaning the immediate neighbourhood of a vertex can be unfolded. This unfoldability propagates throughout the mesh, provided the base mesh is simply connected and vertices have valence 4 . The fact that existence of an unfolding is expressed via angles, has an interesting consequence:

If such a checkerboard kirigami is modified such that combinatorics is maintained, and such that edges remain parallel to their original state, then the kirigami property (existence of an unfolding) is preserved. Borrowing a term from discrete differential geometry [Bobenko and Suris 2009], this means that this class of checkerboard kirigami is closed under Combescure transforms.

3.3.3 Programming curvature and the Theorema Egregium. By Gauss' theorema egregium, Gaussian curvature is unchanged under isometric deformations and is uniquely determined by the intrinsic metric of a surface. For an orthogonal parametrization $f(u, v)$ with $a=\left\|f_{u}\right\|$ and $b=\left\|f_{v}\right\|$ (subscripts indicate derivatives) we have $a b K=\left(b_{u} / a\right)_{u}+\left(a_{v} / b\right)_{v}$, see [do Carmo 1976, p. 237].

The base meshes used in this paper are seen as samples of such a parametrization at intervals $\Delta u$ resp. $\Delta v$. So the edgelengths $\ell_{1}, \ell_{2}$ of $B_{M}$ in the discrete $u$ and $v$ directions can be interpreted as $a \approx \frac{1}{\Delta u} \ell_{1}$ resp. $b \approx \frac{1}{\Delta v} \ell_{2}$. From this, we get $b_{u} \approx \frac{1}{\Delta u \Delta v} \Delta_{1} \ell_{2}$, where $\Delta_{1}$ and $\Delta_{2}$ refer to the increment in the discrete $u$ and discrete $v$ directions. Further, $b_{u} / a \approx \frac{1}{\Delta v} \Delta_{1} \ell_{2} / \ell_{1}$ and $\left(b_{u} / a\right)_{u} \approx \frac{1}{\Delta u \Delta v} \Delta_{1}\left(\Delta_{1} \ell_{2} / \ell_{1}\right)$. With the analogous expression for $\left(a_{v} / b\right)_{v}$ we arrive at

$$
\ell_{1} \ell_{2} K \approx \Delta_{1}\left(\Delta_{1} \ell_{2} / \ell_{1}\right)+\Delta_{2}\left(\Delta_{2} \ell_{1} / \ell_{2}\right) .
$$

For a checkerboard kirigami structure, assuming fairness, the quads on top of boxes are of the same size as the quads in the base mesh those boxes are attached to. In consequence, edgelengths $\ell_{1}, \ell_{2}$ can be read off the unfolding, and so can Gaussian curvature, cf. Fig. 9.

\subsection{Special cases and the Gauss-Bonnet Bonnet formula}

A natural definition of Gauss curvature concentrated in a vertex of a polyhedral surface is $2 \pi$ minus the angle sum in that vertex [Cohen-Steiner and Morvan 2003]. Box-based kirigami structures 


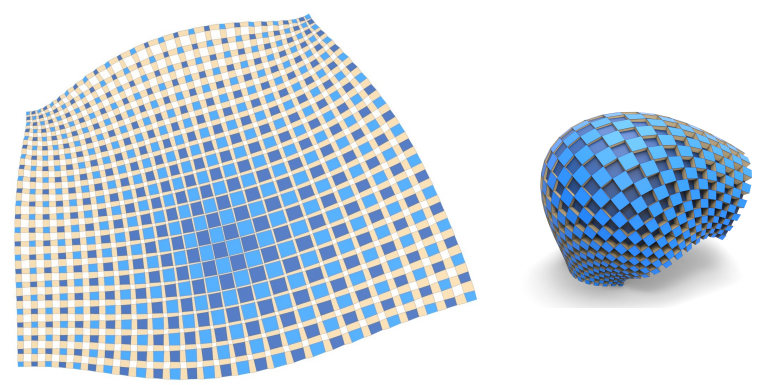

Fig. 9. The Gaussian curvature $K$ of a checkerboard kirigami surface can be computed from edgelengths, the approximate theorema egregium (3) giving a quantitative meaning to the words programming curvature sometimes used in connection with kirigami. E.g. that part of the unfolding where the blue colour dominates has $K>0$, because of a local maximum of edgelengths.

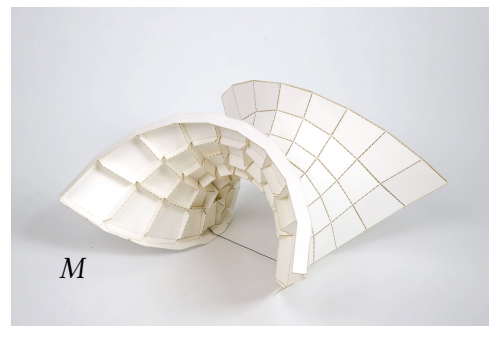
face formed by the tops of boxes display Gaussian curvature in their unfolding: The sum of signed angles $\omega_{i}$ in gaps equals the total Gauss curvature of a surface patch, lending a quantitative meaning to the words programming curvature.
Fig. 10. Kirigami enjoying a seamless sur-

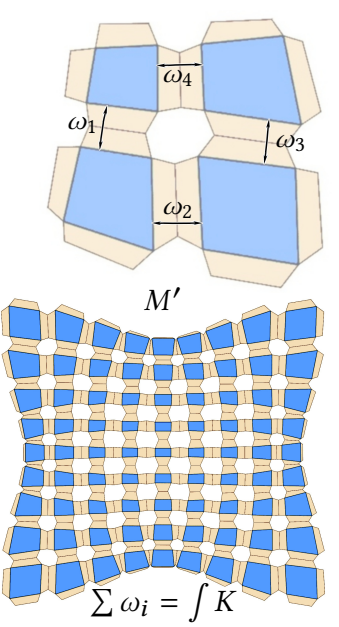

where the tops of boxes form a seamless quad mesh allow a direct application. Examples are provided by the support structure kirigami of Fig. 4 and the generalized support structure kirigami of Fig. 10 where a less strict condition is enforced (namely, each box must share a common side-face with its neighbours). Obviously, the sum of angles $\omega_{i}$ shown in Fig. 10 equals the Gauss curvature concentrated in the central vertex, if we use the convention that the angle $\omega_{i}$ is positive if the gap it occupies opens up then traveling away from the central vertex.

By summation, an analogous formula holds for rectangular patches of $M \times N$ faces. The sum of signed gap angles along the boundary of the unfolding equals the total Gauss curvature concentrated in the interior of the patch, This statement is reminiscent of the local Gauss-Bonnet formula [do Carmo 1976, p. 269]. The symmetries

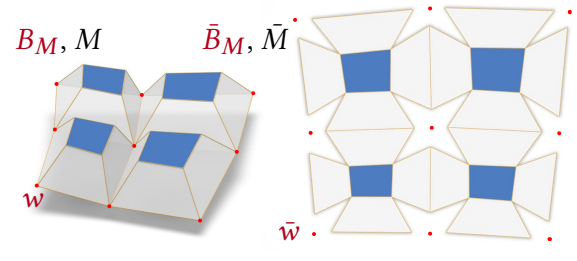

Fig. 11. Base meshes $B_{M}, \bar{B}_{M}$ associated with a kirigami $M$ and its unfolding $\bar{M}$. The vertices of $\bar{B}_{M}$ sit in the centers of holes. present in the unfolding imply that in the spatial kirigami structure, angles $\frac{1}{2} \omega_{i}$ can be observed.

\section{COMPUTING KIRIGAMI STRUCTURES}

The computation of a kirigami structure $M$ together with its unfolding $\bar{M}$ relies on base meshes $B_{M}, \bar{B}_{M}$. We already defined the relation between $B$ and $M$. As to the base mesh $\bar{B}_{M}$, it is combinatorially equivalent to $B$ and has vertices in the holes of the unfolding $\bar{M}$, see Figure 11.

$\S 4$ is devoted to our computational pipeline. It starts with a cross field resp. orthogonal curve network on a reference surface $\Phi$, proceeds to the base mesh $B_{M}$, the base mesh $\bar{B}_{M}$, initial guesses at $M, \bar{M}$, and finally to the computation of $M, \bar{M}$ by optimization:

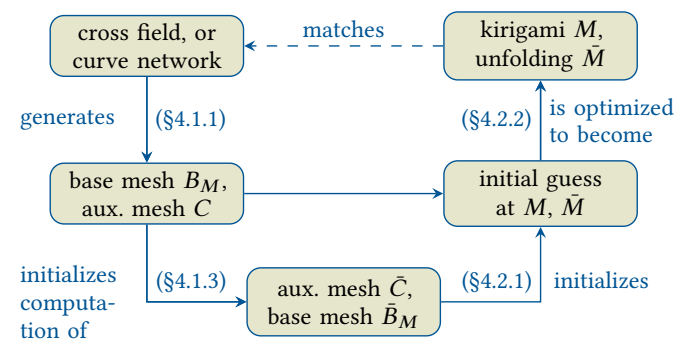

\subsection{Setting up base meshes of kirigami structures}

4.1.1 Base meshes from curve networks. We are generally initializing the base mesh $B_{M}$ as a quad mesh following an orthogonal network of curves on a smooth surface $\Phi$. Equivalently it can be aligned with a smooth cross field [Bommes et al. 2009].

In some cases special curves are required, e.g. principal curvature lines are necessary to produce checkerboard kirigami, see §3.3.1. Otherwise the orthogonality requirement is for reasons of aesthetics, and the choice of curve network is a design decision.

For kirigami based on a checkerboard colouring of the base mesh, even valences are required. In that case the curve network guiding $B_{M}$ can have only singularities whose index is an integer multiple of $1 / 2$. Principal curvature lines enjoy this property provided umbilics are isolated, see e.g. [Cazals and Pouget 2004]. For general box kirigami there are no restrictions on the indices, cf. Figure 12.

Orthogonal networks of curves can be produced easily by conformal parametrizations, but otherwise conformal mappings do not play a role in our work. This is different for the kirigami structures based on hexagonal combinatorics proposed by Konaković et al. [2016; 2018].

4.1.2 Base mesh correspondence as a discrete expanding mapping between curve networks. The next step is to construct the base mesh $\bar{B}_{M}$ associated with the unfolding $\bar{M}$. Since we wish to construct boxes that are as symmetric as possible, also the edges of $\bar{B}_{M}$ are approximately orthogonal to each other. The mapping $B_{M} \rightarrow \bar{B}_{M}$ then discretizes a smooth mapping $\psi: \Phi \rightarrow \mathbb{R}^{2}$ which transforms the given curve network on $\Phi$ to an orthogonal curve network in the plane. These networks are thus aligned with the directions of minimum and maximum distortion of the mapping. 


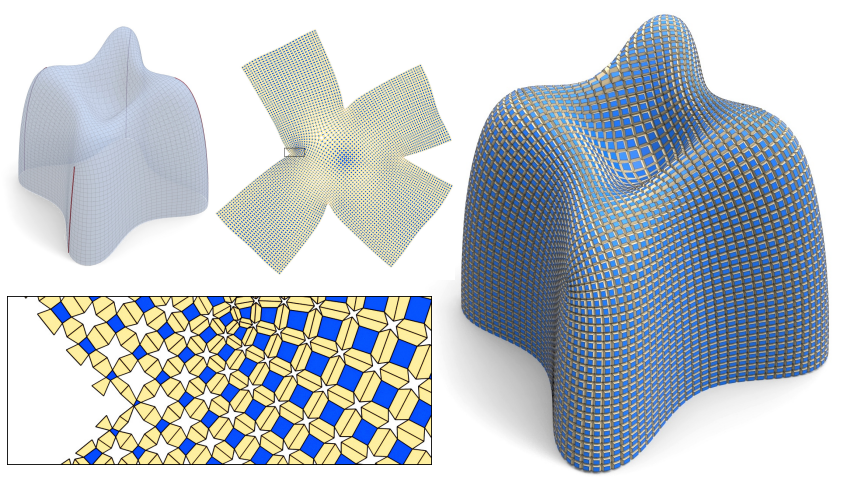

Fig. 12. Cuts enable us to extend our mapping-based method of initializing box kirigami to more complex geometries, while keeping distortions small.

Since the mapping $\psi$ corresponds to the unfolding of boxes, it is expanding, with principal distortions $\geq 1$. The limit case of an exact isometry, which corresponds to paper folding without cutting, has principal distortions equal to 1 . While box-based kirigami structures can simulate mappings with quite high distortions, it is nevertheless advisable to make use of the freedom offered by cutting open the design surface and achieve lower distortion. This in turn makes box heights smaller. Figure 12 shows an example of this. Such cuts are invisible in the final kirigami structure but are, of course, very much visible in the unfolding

4.1.3 Computing the base mesh correspondence. For computing the discrete mapping $B_{M} \rightarrow \bar{B}_{M}$ it is convenient to employ a trick motivated by [Jiang et al. 2019b]: The inset figure below explains how the edges of $B_{M}$ can be expressed as one half of the diagonals of an auxiliary mesh $C$ (yellow). An analogous relation holds between $\bar{B}_{M}$ and a corresponding auxiliary mesh $\bar{C}$. With this setup, we do the following.

- Define the mesh $C$ according to the combinatorics specified above. Each vertex $w_{i}$ of $B_{M}$ will be a vertex of $C$. For each face $f_{l}$ of $B_{M}$, add its center of mass as a vertex $w_{l}^{\text {face }}$ of $C$.

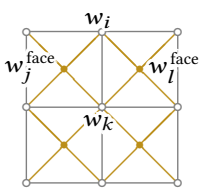

- Set up a mesh $\bar{C}$ combinatorially equivalent to $C$. The combinatorial relation between $\bar{C}$ and $\bar{B}_{M}$ is analogous to the relation between $C$ and $B_{M}$. For the computation of vertices of $\bar{C}, \bar{B}_{M}$, recall the relation between the base mesh $\bar{B}_{M}$ and the unfolding, see Fig. 11. Passing from $B_{M}$ to $\bar{B}_{M}$ corresponds to unfolding the boxes glued into faces. All faces become larger in that process, see Fig. 13 for a computation regarding very symmetric boxes.

- We model the expansion of a face $f_{l}$ in an extremely simple manner. We think of it growing on all four sides by the same nonnegative amount $\eta_{l}^{2}$. Consequently, an edge $w_{i} w_{k}=f_{j} \cap f_{l}$ of the base mesh causes associated diagonals of the auxiliary mesh to expand. These are $w_{i} w_{k}$ (the edge itself) and $w_{j}^{\text {face }} w_{l}^{\text {face }}$ (across the edge). Their expansion obeys

$$
\begin{gathered}
\left\|w_{j}^{\text {face }}-w_{l}^{\text {face }}\right\|+\eta_{j}^{2}+\eta_{l}^{2}-\left\|\bar{w}_{j}^{\text {face }}-\bar{w}_{l}^{\text {face }}\right\|=0, \\
\left\|w_{i}-w_{k}\right\|+\eta_{j}^{2}+\eta_{l}^{2}-\left\|\bar{w}_{i}-\bar{w}_{k}\right\|=0 .
\end{gathered}
$$

Equ. (4) is only a rough approximation of the true expansion of the boxes contained in a kirigami structure. This does not matter at this stage, since our only goal is to compute an initial guess at $\bar{B}_{M}$, to be optimized later.

For the checkerboard kinds of kirigami, where only half of the base mesh's faces are replaced by boxes, we let $\eta_{l}^{2}=0$ whenever the face $f_{l}$ stays flat.

- In addition we require that the edges of $\bar{B}_{M}$ are approximately orthogonal to each other. We do this by requiring diagonals of $C$ to be exactly orthogonal:

$$
\left(\bar{w}_{i}-\bar{w}_{k}\right)^{T} \cdot\left(\bar{w}_{j}^{\text {face }}-\bar{w}_{l}^{\text {face }}\right)=0 .
$$

In this way we never actually impose strict orthogonality between any edges of $\bar{B}_{M}$, and we also avoid to specify an approximate version of orthogonality. Instead we leverage the additional degrees of freedom provided by the auxiliary mesh $\bar{C}$.

- The actual computation of $\bar{B}_{M}$ is done by optimization. We create an initial guess using the ARAP method [Liu et al. 2008; Sorkine and Alexa 2007] and optimize an energy which is the sum, over all edges, of squares of constraints (4) and (5). The optimization method is the one of $§ 4.2 .2$.

\subsection{Building kirigami from a base mesh pair}

4.2.1 Initializing optimization. Having established a preliminary correspondence of base meshes $B_{M}, \bar{B}_{M}$, we need to construct initial guesses at the kirigami structure $M$ and its unfolding $\bar{M}$, reversing the process which for a simple box geometry is shown by Fig. 13 . For any face $f_{j}$ of the base mesh $B$, the variable $\eta_{j}^{2}$ specifies how much it extends. The formula of Fig. 13 then yields a set of valid values for box height $h$ and inclination angle $\beta$. We are still in the stage of initializing optimization, so we construct only simple boxes whose top is parallel to the bottom (so their height is well defined). For most examples in this paper, the choice of box height is guided by a point dependent factor $s_{f}$ indicating the ratio between the size of the top of the box to the size of the bottom face. We can go from $s_{f}=0$ as in Fig. 20 to $s_{f}=1$ as in Fig. 4, and we can make $s_{f}$ face-dependent as in Fig. 18. In some examples, the minimum feasible height was used, like in Fig. 15. These parameters $s_{f}$ are chosen by the user.

We also initialize $\bar{M}$ by deforming the regular unfolding of Fig. 13 so that it fits the given faces of $\bar{B}_{M}$. As it turns out, this crude method of initializing $B_{M}, \bar{B}_{M}$ is sufficient.

4.2.2 Global optimization of kirigami structures and unfoldings. We start this section by setting down conditions meshes $M, \bar{M}$ have to satisfy to be a kirigami structure and its unfolding. We are going to meet these constraints by global optimization.

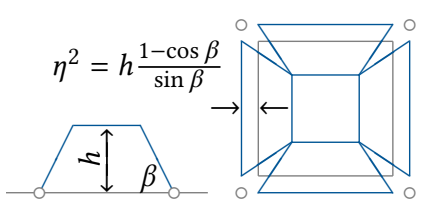

Fig. 13. Unfolding a symmetric box glued into the face of a base mesh $B_{M}$. The unfolded box is bigger by an amount computable from height and inclination angle of the box. 

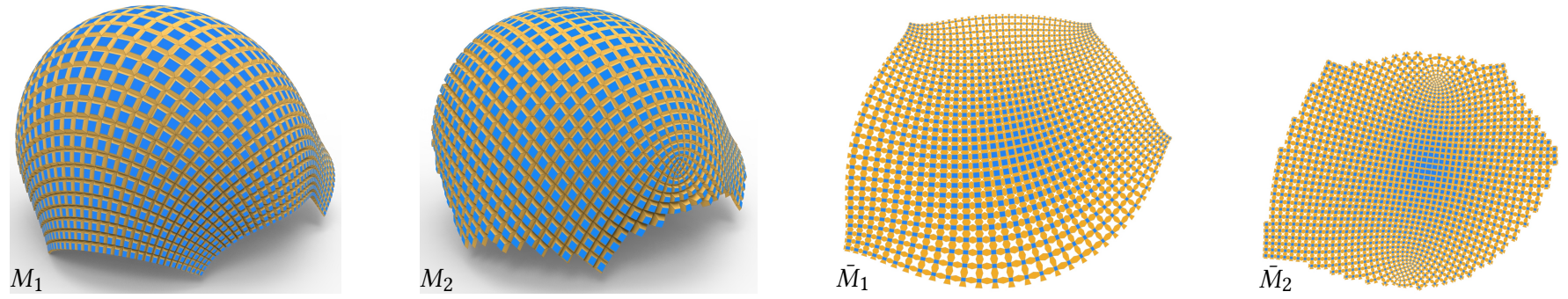

Fig. 14. Efficient use of material. The kirigami $M_{1}$ is improved by remeshing along the principal directions of distortion of an as-rigid-as-possible mapping to the plane. This yields a kirigami $M_{2}$ whose unfolding is smaller than $\bar{M}_{1}$ and has smaller holes, due to the fact that ARAP mappings have distortions close to 1 (the ARAP method is used anyway in our computing pipeline, but here it is additionally used to create a cross field which initializes the base mesh $B_{M}$ ).

Our setup, described above, is a pair of combinatorially equivalent quad meshes $M, \bar{M}$ with planar faces, and $\bar{M}$ contained in $\mathbb{R}^{2}$ Corresponding faces of $M, \bar{M}$ are supposed to be isometric, which is expressed by equality of lengths of corresponding edges and diagonals. These equalities are true if and only if the energy

$$
E_{\mathrm{iso}}(M, \bar{M})=\sum_{f \in F} \sum_{v, w \in f}\left(\|v-w\|^{2}-\|\bar{v}-\bar{w}\|^{2}\right)^{2}
$$

evaluates to zero. Here the symbols $\bar{v}, \bar{w}$ indicate the vertices of $\bar{M}$ corresponding to the vertices $v, w$ of $M$.

Note that the isometry constraints already imply planarity of the faces of $M$ (since the faces of $\bar{M}$ are planar by default). It turns out that also in practice, our optimization produces sufficiently planar faces, and no further constraints expressing planarity are needed.

For the closing of holes present in $\bar{M}$, we define a list $G$ of pairs of vertices in $M$ which are required to be in the same position. Typically $50 \%$ of the vertices of a hole are required to coincide. Also cuts made in the initialization phase (e.g. in Fig. 12) are dealt with by this list. Vertex positions coincide if and only if the following energy is zero:

$$
E_{\text {close }}(M)=\sum_{(v, w) \in G}\|v-w\|^{2} .
$$

Regularization. In view of the applications we have in mind, we also need a way to express fairness. It can be conveniently expressed in terms of fairness of the base meshes $B_{M}$ and $\bar{B}_{M}$, thus implicitly penalizing uneven dimensions of boxes:

$$
E_{\mathrm{fair}}(M, \bar{M})=\sum_{\substack{w_{i} w_{j} w_{k} \text { succes- } \\
\text { ive vertices of } B_{M}}}\left\|w_{i}-2 w_{k}+w_{k}\right\|^{2}+\sum_{\begin{array}{c}
\bar{w}_{i} \bar{w}_{j} \bar{w}_{k} \text { succes- } \\
\text { ive vertices of } \bar{B}_{M}
\end{array}}\left\|\bar{w}_{i}-2 \bar{w}_{j}+\bar{w}_{k}\right\|^{2} .
$$
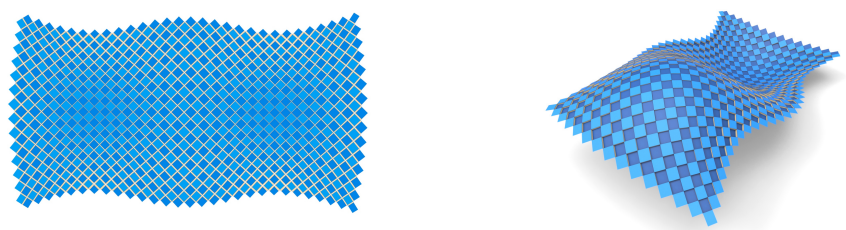

Fig. 15. Efficient use of material. By using boxes of small height we achieve a type $C$ kirigami structure where only a small area of the unfolding contributes to the boxes' sides. This kirigami is initialized from the network of principal curvature lines of an isothermic surface, which means quads are close to squares, cf. [Bobenko and Suris 2009].
The sum is over all triples of successive vertices of mesh polylines. In case the base mesh has been cut open as in Fig. 12, fairness of $M$ (not of $\bar{M}$ ) is also enforced across the cuts.

The optimization algorithm. Summing up, we compute the kirigami structure $M$ by determining vertices of $M, \bar{M}$ such that

$$
E=E_{\text {iso }}+E_{\text {close }}+w_{\text {fair }} E_{\text {fair }}
$$

is minimal. Here $w_{\text {fair }}$ is a small weight. This energy is minimized by a Levenberg-Marquardt method according to [Madsen et al. 2004, §3.2], using a damping parameter of $10^{-6}$. We should add that we could just as well have used other optimization methods. We performed a fixed number of 10 iterations. Our implementation in $\mathrm{C}++$ uses the data structures of OpenMesh [Botsch et al. 2002] and the TAucs library for sparse linear solvers [Toledo 2003].

Detailed statistics are provided by the table in Fig. 17. The computation times refer to an Intel Xeon E5-2687W 3.0 GHz processor without parallel processing or other acceleration techniques.

\section{DISCUSSION}

\subsection{Examples}

We start by giving some examples how the methods described above can be used and generalized. Several such examples are contained in the explanatory figures in previous sections.

Kirigami with specific shapes of boxes. It should be emphasized that the initialization of $M, \bar{M}$ in $\S 4.2 .1$ can be used to model the appearance of boxes, as shown by Fig. 18. A more drastic method to define a specific shape is to use constraints to achieve that all four vertices on top of each box coincide, see Fig. 20.

Kirigami with efficient use of material. Small heights of boxes means that the holes to be cut out in the unfolding are small. Fig 15 shows that this is possible even with a highly constrained type $\mathrm{C}$ kirigami structure. The low heights are firstly achieved by adding the energy $\sum_{f_{l} \in F} \eta_{l}^{2}$ (with a low weight) in the optimization procedure which produces the base mesh correspondence in $\S 4.1 .3$, and secondly using the smallest heights which are possible in the initialization procedure of $\S 4.2 .1$.

Remeshing according to principal distortions. The heights of boxes and size of holes directly correspond to the expansion factor in the correspondence between base meshes $B_{M}$ and $\bar{B}_{M}$. If that mapping is close to isometric, factors are close to 1 and box heights are small. 

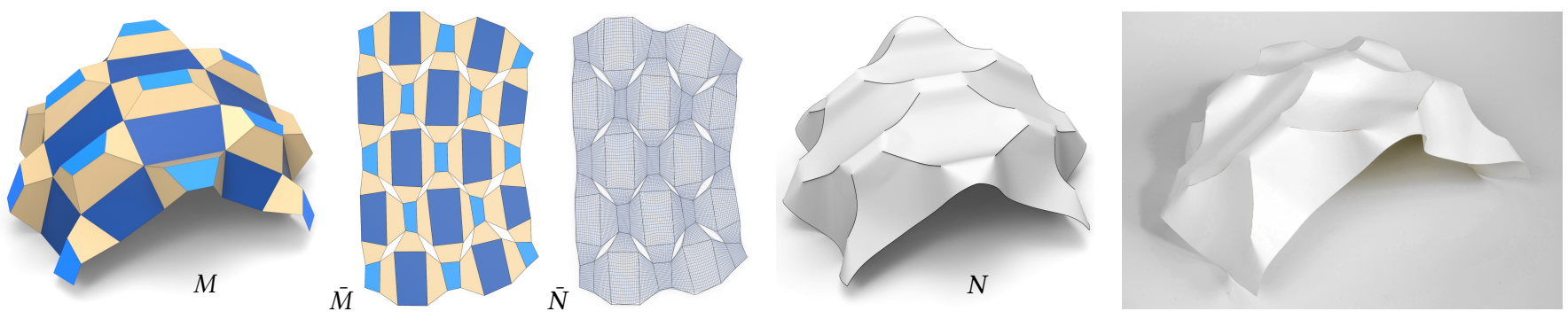

Fig. 16. A coarse CB kirigami structure $M$ is modified by applying subdivision to its unfolding $\bar{M}$, creating a planar domain $\bar{N}$ exhibiting holes with curved boundaries. We close these holes in an isometric way, simulating gluing. The resulting piecewise-developable surface $N$ has been created by the method of [Jiang et al. 2020]. The figure at right shows a paper model.

\begin{tabular}{ccccccccc} 
Fig. & $\left|V_{B}\right|$ & $\left|V_{M}\right|$ & $\left|F_{M}\right|$ & $w_{\text {fair }}$ & T & L-Err & Pl-Err (max/av.) & Pr-Err (max/av.) \\
\hline 1 & $1.4 \mathrm{k}$ & $10 \mathrm{k}$ & $6.3 \mathrm{k}$ & $1 \mathrm{E}-4$ & 17 & $3.7 \mathrm{E}-6$ & $6.6 \mathrm{E}-4 / 2.1 \mathrm{E}-4$ & $3.4 \mathrm{E}-4 / 5.3 \mathrm{E}-5$ \\
2 & $0.44 \mathrm{k}$ & $3.9 \mathrm{k}$ & $2.3 \mathrm{k}$ & $1 \mathrm{E}-4$ & 3.7 & $5.9 \mathrm{E}-6$ & $9.2 \mathrm{E}-4 / 2.3 \mathrm{E}-4$ & $1.1 \mathrm{E}-3 / 7.3 \mathrm{E}-5$ \\
$7 \mathrm{a}$ & $0.45 \mathrm{k}$ & $3.2 \mathrm{k}$ & $1.9 \mathrm{k}$ & $1 \mathrm{E}-4$ & 3.2 & $2.6 \mathrm{E}-6$ & $4.0 \mathrm{E}-4 / 1.7 \mathrm{E}-4$ & $6.1 \mathrm{E}-4 / 1.1 \mathrm{E}-4$ \\
$7 \mathrm{~b}$ & $0.45 \mathrm{k}$ & $3.2 \mathrm{k}$ & $1.9 \mathrm{k}$ & $1 \mathrm{E}-4$ & 4.5 & $9.2 \mathrm{E}-7$ & $2.2 \mathrm{E}-5 / 3.5 \mathrm{E}-6$ & $2.7 \mathrm{E}-3 / 5.5 \mathrm{E}-4$ \\
$7 \mathrm{c}$ & $0.45 \mathrm{k}$ & $1.5 \mathrm{k}$ & $1.0 \mathrm{k}$ & $1 \mathrm{E}-4$ & 1.1 & $3.7 \mathrm{E}-6$ & $6.8 \mathrm{E}-4 / 1.9 \mathrm{E}-4$ & $6.1 \mathrm{E}-3 / 5.7 \mathrm{E}-4$ \\
9 & $0.67 \mathrm{k}$ & $2.5 \mathrm{k}$ & $1.8 \mathrm{k}$ & $1 \mathrm{E}-2$ & 3.8 & $3.4 \mathrm{E}-5$ & $3.0 \mathrm{E}-4 / 4.9 \mathrm{E}-5$ & $3.1 \mathrm{E}-3 / 3.1 \mathrm{E}-4$ \\
12 & $5.1 \mathrm{k}$ & $50 \mathrm{k}$ & $25 \mathrm{k}$ & $1 \mathrm{E}-4$ & 115 & $4.9 \mathrm{E}-6$ & $3.0 \mathrm{E}-4 / 1.1 \mathrm{E}-4$ & $1.2 \mathrm{E}-3 / 5.4 \mathrm{E}-5$ \\
14 & $1.5 \mathrm{k}$ & $11 \mathrm{k}$ & $6.9 \mathrm{k}$ & $1 \mathrm{E}-4$ & 29 & $1.6 \mathrm{E}-5$ & $3.2 \mathrm{E}-4 / 4.9 \mathrm{E}-5$ & $2.3 \mathrm{E}-3 / 2.2 \mathrm{E}-4$ \\
15 & $1.9 \mathrm{k}$ & $3.4 \mathrm{k}$ & $2.4 \mathrm{k}$ & $1 \mathrm{E}-2$ & 5.3 & $1.3 \mathrm{E}-6$ & $2.7 \mathrm{E}-4 / 6.6 \mathrm{E}-5$ & $1.6 \mathrm{E}-3 / 1.5 \mathrm{E}-4$ \\
18 & $0.40 \mathrm{k}$ & $2.9 \mathrm{k}$ & $1.8 \mathrm{k}$ & $1 \mathrm{E}-4$ & 3.0 & $3.4 \mathrm{E}-6$ & $6.2 \mathrm{E}-4 / 1.9 \mathrm{E}-4$ & $2.5 \mathrm{E}-4 / 1.1 \mathrm{E}-4$ \\
21 & $1.3 \mathrm{k}$ & $10 \mathrm{k}$ & $6.3 \mathrm{k}$ & $1 \mathrm{E}-4$ & 15 & $1.1 \mathrm{E}-5$ & $2.2 \mathrm{E}-4 / 3.6 \mathrm{E}-5$ & $1.0 \mathrm{E}-3 / 7.6 \mathrm{E}-4$ \\
22 & $2.3 \mathrm{k}$ & $17 \mathrm{k}$ & $10 \mathrm{k}$ & $1 \mathrm{E}-4$ & 44 & $3.8 \mathrm{E}-6$ & $3.2 \mathrm{E}-4 / 1.8 \mathrm{E}-4$ & $1.5 \mathrm{E}-3 / 5.9 \mathrm{E}-5$
\end{tabular}

Fig. 17. Optimization statistics. We show the size of base mesh $B_{M}$ and kirigami structure $M$, the weight $w_{\text {fair }}$, and the time in seconds needed for optimization. The quality of the isometric relation between $M$ and its unfolding $\bar{M}$ is measured via the relative $L^{2}$ error of edgelengths, $\| \bar{L}-$ $L\|/\| L \|$. Here $L, \bar{L}$ refer to the vector of edgelengths of meshes $M, \bar{M}$, and $\|\cdot\|$ is the Euclidean norm. The column "PI-Err" shows the maximal/average deviation from planarity of faces; the figure given refers to the distance between diagonals of quadrilateral faces divided by average edge length. The last column illustrates maximal/average deviation of the base vertices from the reference surface (as a fraction of the bounding box diameter).

We can therefore achieve low box heights by choosing an as-rigid-aspossible correspondence [Liu et al. 2008; Sorkine and Alexa 2007]. The singular value decomposition of a local linearization of the mapping yields the directions of minimal and maximal distorsions. We use the libigl implementation of mixed-integer quadrangulation [Bommes et al. 2009; Jacobson et al. 2018] to find a quad mesh $B_{M}$ aligned with this cross field and proceed with optimization from there. Fig. 14 shows an example.

\subsection{Extensions}

The kirigami principle employed so far can be generalized and extended. One extension is the passage to curved cuts. Figure 16 gives an example where the unfolding $\bar{M}$ of a box kirigami structure undergoes Catmull-Clark subdivision modified such that for each top of a box, the four vertices stay fixed. We subsequently use the method of [Jiang et al. 2020] to find a surface in space isometric to this modified unfolding, such that opposite edges of holes are glued together. Examples of this kind are already shown by Jiang et al.
[2020], the difference to our work being that we can prescribe the shape of the final surface as well as the pattern of cuts.

Another extension of our basic principle for generating kirigami is a generalization of our kirigami support structures as explained by Figure 19. An example was already contained in Fig. 10.

\subsection{Limitations and Future Research}

- An obvious limitation is that the distortions of the mapping between base meshes must be implicitly encoded in the dimensions of the boxes which make up the kirigami structure. The extent of distortions we can handle is demonstrated by means of the examples in this paper e.g. Fig. 21. If distortions are too large, or the two principal distortions are too different from each other, the method can fail. Figure 12 would already have been a fail case without cutting. Another fail case are collisions between boxes when we try to model sharp concave features.

- A limitation on the theoretical side is that due to the nonconvex nature of optimization, success of our procedures cannot

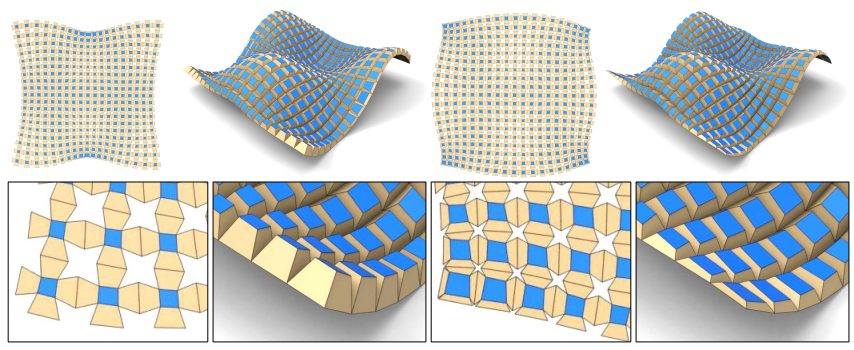

Fig. 18. Kirigami with differently shaped boxes. Optimization has been started from the same base mesh pair, but a different initialization of boxes obviously impacts the shape of the unfolding.

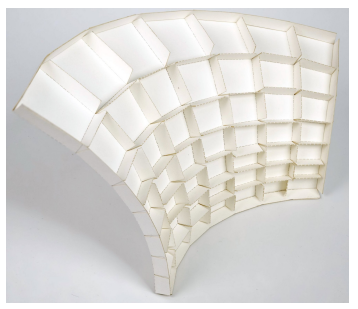

Fig. 19. A generalized support structure. For each edge of the base mesh, the two adjacent boxes are required to share a face. The holes present in the unfolding close in a manner different from other box-based kirigami structures. The sides of boxes form flaps useful for model building. 

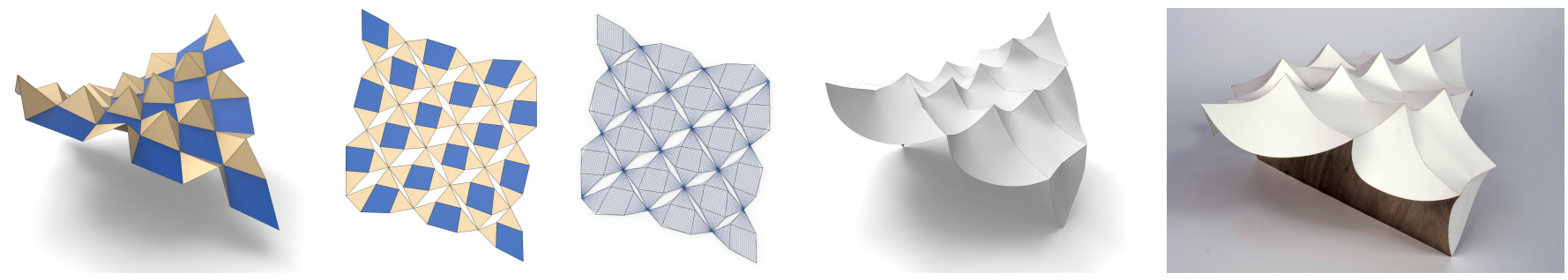

Fig. 20. This CB kirigami structure features pyramid boxes with degenerate top face. In the unfolding, holes touch each other. The unfolding has been modified by applying subdivision, creating a planar domain with curved holes. By closing these holes, with the method of [Jiang et al. 2020], we create a piecewise-developable surface. Compared to previous work, the main advance of the present paper is that we can design both the shape of this curved-crease kirigami as well as the pattern of cuts.

be guaranteed mathematically. Planarity of faces, isometry to a $2 \mathrm{D}$ domain, and absence of collisions can only be verified a posteriori.

- We also did not discuss the possibility of a continuous isometric folding path from the flat to the final state. This is a particularly interesting question and would be highly relevant to transformable design in architecture.

- An important topic of further future research, closely linked to the success guarantees mentioned above, is existence. While type B kirigami seems not too difficult, this is different for type $\mathrm{C}$ and support structure kirigami. Numerical experiments confirm existence, but the explicit construction of nontrivial special cases does not appear to be easy.

- Further connections to discrete differential geometry, e.g. on the meaning of mean curvature, would be very interesting.

\subsection{Physical Properties and Fabrication}

$\S 1$ already discussed some aspects of fabrication on the small and medium scale. We did some practical experiments ourselves with box kirigami structures of different types made from paper (Figures $4,10,16,19,20,23$ ) and from plywood (Fig. 24).

As to physical properties of spatial kirigami structures, we experienced unexpectedly high stiffness of type $C$ kirigami like the one in Fig. 23, which also goes hand in hand with very small error tolerances in manufacturing. We believe this occurs because the arrangement of quads in a type $\mathrm{C}$ kirigami prevents buckling in faces.
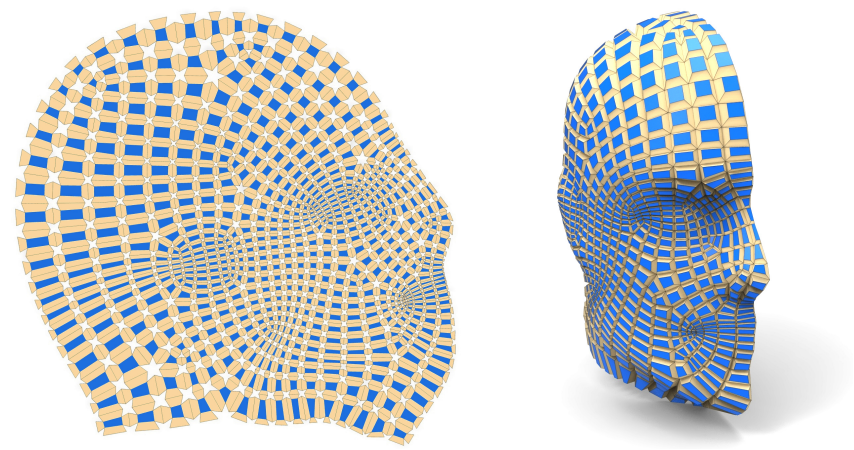

Fig. 21. Here one half of a head is represented as a box kirigami structure. A reflected copy completes a kirigami representation of the entire head.
We are also envisioning kirigami made from sheet metal whose actual fabrication however was beyond the authors' capabilities. Any structure should be feasible as long as folding can be done manually and the sheet can be handled by two persons.

\section{ACKNOWLEDGMENTS}

This work was supported by the SFB-Transregio programme Discretization in geometry and dynamics, through grant I2978 of the Austrian Science Fund, and the WWTF under grant ICT15-082. Caigui Jiang and Florian Rist were supported by KAUST baseline funding.

\section{REFERENCES}

Alexander Bobenko and Yuri Suris. 2009. Discrete differential geometry: Integrable Structure. Number 98. American Math. Soc.

David Bommes, Henrik Zimmer, and Leif Kobbelt. 2009. Mixed-integer Quadrangulation. ACM Trans. Graph. 28, 3 (2009), \#77,1-10.

Mario Botsch, Stephan Steinberg, Stephan Bischoff, and Leif Kobbelt. 2002. OpenMesh: A Generic and Efficient Polygon Mesh Data Structure. Proc. OpenSG Symposium.

Toen Castle, Yigil Cho, Xingting Gong, Euiyeon Jung, Daniel M. Sussman, Shu Yang, and Randall D. Kamien. 2014. Making the Cut: Lattice Kirigami Rules. Phys. Rev. Lett 113, 245502 (2014), 1-5.

Toen Castle, Daniel M. Sussman, Michael Tanis, and Randall D. Kamien. 2016. Additive lattice kirigami. Sci. Advances 2, e1601258 (2016), 1-11.

Frédéric Cazals and Marc Pouget. 2004. Smooth surfaces, umbilics, lines of curvatures, foliations, ridges and the medial axis: a concise overview. Research Report RR-5138. INRIA. https://hal.inria.fr/inria-00071445/

Gary Choi, Levi Dudte, and L. Mahadevan. 2019. Programming shape using kirigami tessellations. Nature Materials 18 (2019), 999-1004.

David Cohen-Steiner and Jean Marie Morvan. 2003. Restricted Delaunay triangulations and normal cycle. In Proc. ACM Symp. Comp. Graphics. 312-321.

Erik D. Demaine and Joseph O'Rourke. 2007. Geometric Folding Algorithms. Cambridge University Press.

Erik D. Demaine and Tomohiro Tachi. 2017. Origamizer: A Practical Algorithm for Folding Any Polyhedron. In Proc. 33rd SoCG. 1-15.

Manfredo do Carmo. 1976. Differential Geometry of Curves and Surfaces. Prentice-Hall.

Levi H. Dudte, Etienne Vouga, Tomohiro Tachi, and L. Mahadevan. 2016. Programming curvature using origami tessellations. Nature Materials 15 (2016), 583-588.

Ruslan Guseinov, Connor McMahan, Jesús Pérez, Chiara Daraio, and Bernd Bickel. 2020. Programming temporal morphing of self-actuated shells. Nature Communications 11 (2020), \#237,1-7.

Ruslan Guseinov, Eder Miguel, and Bernd Bickel. 2017. CurveUps: shaping objects from flat plates with tension-actuated curvature. ACM Trans. Graph. 36, 4 (2017), $\# 64,1-12$.

Alec Jacobson, Daniele Panozzo, et al. 2018. libigl: A simple C++ geometry processing library. https://libigl.github.io

Caigui Jiang, Klara Mundilova, Florian Rist, Johannes Wallner, and Helmut Pottmann. 2019a. Curve-pleated structures. ACM Trans. Graph. 38, 6 (2019), \#169,1-13.

Caigui Jiang, Chi-Han Peng, Peter Wonka, and Helmut Pottmann. 2019b. Checkerboard patterns with black rectangles. ACM Trans. Graph. 38, 6 (2019), \#171,1-13.

Caigui Jiang, Cheng Wang, Florian Rist, Johannes Wallner, and Helmut Pottmann. 2020. Quad-mesh based isometric mappings and developable surfaces. ACM Trans. Graph. 39, 4 (2020), \#128,1-13. 

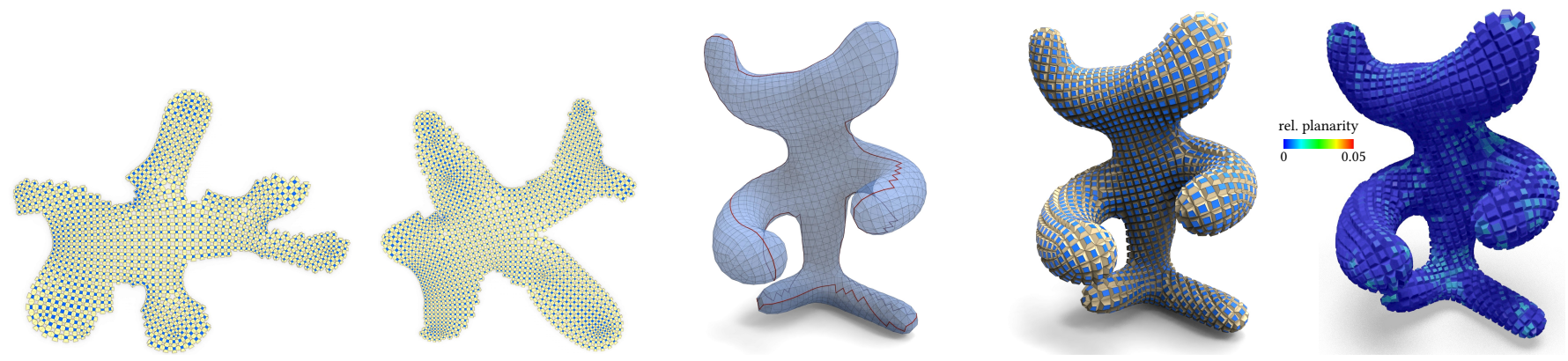

Fig. 22. A cross field on the reference surface defines a quad mesh, and by cutting the mesh along a closed mesh polyline we achieve low-distortion mappings into the plane that can initialize a box-based kirigami structure approximating the original cross field. That cross field has been designed using the libigl implementation of the method of [Ray et al. 2008]. The image at right shows planarity of faces, cf. the table in Fig. 17.

Mina Konaković, Keenan Crane, Bailin Deng, Sophien Bouaziz, Daniel Piker, and Mark Pauly. 2016. Beyond Developable: Computational Design and Fabrication with Auxetic Materials. ACM Trans. Graph. 35, 4 (2016).

Mina Konaković-Luković, Julian Panetta, Keenan Crane, and Mark Pauly. 2018. Rapid Deployment of Curved Surfaces via Programmable Auxetics. ACM Trans. Graph.37, 4 (2018), \#106,1-13.

Ligang Liu, Lei Zhang, Yin Xu, Craig Gotsman, and Steven Gortler. 2008. A local/global approach to mesh parameterization. Comput. Graph. Forum 27, 5 (2008), 1495-1504.

Kaj Madsen, Hans Bruun Nielsen, and Ole Tingleff. 2004. Methods for non-linear least squares problems (2nd ed.). Technical Univ. Denmark.

Tanmoy Mukhopadhyay et al. 2020. Programmable stiffness and shape modulation in origami materials: emergence of a distant actuation feature. Applied Materials Today 19, 100537 (2020), 1-7.

Helmut Pottmann, Yang Liu, Johannes Wallner, Alexander Bobenko, and Wenping Wang. 2007. Geometry of Multi-layer Freeform Structures for Architecture. ACM Trans. Graph. 26, 3 (2007), \#65,1-11.

Nicolas Ray, Bruno Vallet, Wan Chiu Li, and Bruno Lévy. 2008. N-Symmetry Direction Field Design. ACM Trans. Graph. 27, 2 (2008), \#10,1-13.

Keith A. Seffen. 2012. Compliant shell mechanisms. Phil. Trans. R. Soc. A 370 (2012), 2010-2026.

Olga Sorkine and Marc Alexa. 2007. As-rigid-as-possible surface modeling. In Proc. Symposium Geometry Processing. 109-116.

Daniel M. Sussman, Yigil Cho, Toen Castle, Xingting Gong, Euiyeon Jung, Shu Yang, and Randall D. Kamien. 2015. Algorithmic lattice kirigami: A route to pluripotent materials. PNAS 112, 24 (2015), 7449-7453.

Tomohiro Tachi. 2009. Generalizationof Rigid Foldable Quadrilateral Mesh Origami. In Proc. IASS Symposium. Univ. Politècnica de València, 2287-2294.

Tomohiro Tachi. 2010a. Freeform Variations of Origami. F. Geom. Graphics 14 (2010), 203-215.

Tomohiro Tachi. 2010b. Origamizing Polyhedral Surfaces. IEEE Trans. Vis. Comput. Graph. 16 (2010), 298-311.

Yichao Tang, Yanbin Li, Yaoye Hong, and Jie Yin. 2019. Programmable active kirigami metasheets with more freedom of actuation. PNAS 116 (2019), 26407-13.

Sivan Toledo. 2003. TAucs, A Library of Sparse Linear Solvers. http://www.tau.ac.il/ stoledo/taucs

Fei Wang, Xiaogang Guo, Jingxian Xu, Yihui Zhang, and C. Q. Chen. 2017. Patterning curved three-dimensional structures with programmable kirigami designs. f. Appl. Mech. 84, 061007 (2017), 1-7.

Zhiyuan Wei, Zengcai V. Guo, Levi Dudte, Hiyi Liang, and L. Mahadevan. 2013. Geometric Mechanics of Periodic Pleated Origami. Phys. Rev. Lett. 110, 215501 (2013), $1-5$.

Ruikang Xie, Yan Chen, and Joseph M. Gattas. 2015. Parametrisation and application of cube and eggbox-type folded geometries. Intl. J. Space Structures 30 (2015), 99-110.

Yihui Zhang et al. 2015. A mechanically driven form of kirigami as a route to 3D mesostructures in micro/nanomembranes. PNAS 112 (2015), 11757-64.

\section{APPENDIX}

Hypothetical box kirigami with nonplanar faces. Our definitions of box kirigami require planar faces. This is necessary, if we want to keep the property that the kirigami is made by cutting and folding a flat sheet along straight lines (which is the most practicable method) The reasoning is as follows. When isometrically mapping a flat cut/fold pattern $\bar{M}$, two faces $\bar{f}_{1}, \bar{f}_{2}$ to either side of a fold $\bar{c}$ become developable surfaces $f_{1}, f_{2}$, while $\bar{c}$ becomes a curve $c$. Another situation that occurs is that two straight boundary segments $\bar{c}_{1}, \bar{c}_{2}$ of faces $\bar{f}_{1}, \bar{f}_{2}$, are isometrically mapped to the same curve $c$ which is the common boundary of developables $f_{1}, f_{2}$. In both cases, $c$ remains a shortest path on $f_{j}, j=1,2$. If $c$ is actually curved, then its osculating plane is orthogonal to $f_{j}$ [do Carmo 1976], implying that tangent planes of $f_{1}, f_{2}$ coincide and there is no fold. The alternative is that $c$ is straight. Since all faces in a box kirigami have four boundaries of the kind described here, and straight lines on non-flat developables are rulings, this is possible only if faces are flat.

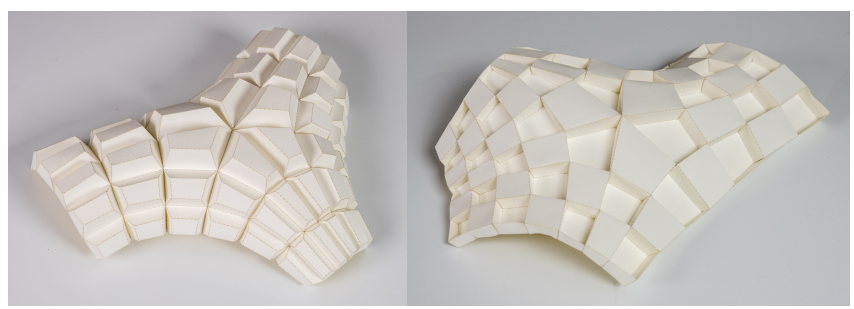

Fig. 23. Box kirigami structures are here made from paper that has been cut and perforated by a laser. We show a type B kirigami (left) and a type $\mathrm{C}$ kirigami (right) which is particularly rigid.
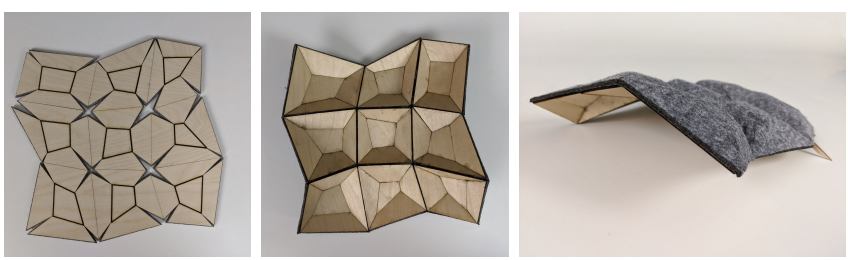

Fig. 24. This small piece of box kirigami has been optimized to periodically fill a horizontal layer of space. It serves as a 1:10 small scale model of a building block for a suspended ceiling for acoustic dampening. We laser cut $1.5 \mathrm{~mm}$ plywood and laminated a pre-cut felt sheet onto it. The width of cuts has been adapted to the angle of the plywood hinges after deployment. 